\title{
Detection of methane at 1670-nm band with a hollow-core photonic bandgap fiber
}

\author{
A. M. Cubillas ${ }^{* a}$, M. Silva-Lopez ${ }^{\mathrm{a}}$, J. M. Lazaro ${ }^{\mathrm{a}}$, O. M. Conde ${ }^{\mathrm{a}}$, M. N. Petrovich ${ }^{\mathrm{b}}$ and J. M. Lopez- \\ Higuera $^{\mathrm{a}}$ \\ ${ }^{a}$ Grupo de Ingeniería Fotónica, Dpto. TEISA, Universidad de Cantabria, Avda. Castros S/N, C.P. \\ 39005 Santander, Spain; \\ ${ }^{b}$ Optoelectronics Research Centre, University of Southampton, Southampton, SO17 1BJ, UK
}

\begin{abstract}
In recent years, hollow-core photonic bandgap fibers (HC-PBFs) have been demonstrated to be a promising technology for gas sensing. In particular, the long interaction path lengths available with these fibers are especially advantageous for the detection of weakly absorbing gases such as methane. In the near-infrared region, methane has the strongest absorption band, $2 v_{3}$, at $1670 \mathrm{~nm}$. However, HC-PBFs were not available until recently in this wavelength range and gas sensing devices based on HC-PBFs were previously made in the weaker band of $1300 \mathrm{~nm}$. In this paper, we report the demonstration of a methane sensor based on a 1670-nm-band HC-PBF. A strong spectral feature, the R(6) manifold (near $1645 \mathrm{~nm}$ ), was selected for sensing purposes as it shows a good signal-to-noise ratio. This absorption line is comprised of six energy transitions, strongly overlapped at our experimental conditions. For that reason, we applied a multiline algorithm that used information from the six transitions to fit the manifold. The goodness of the fitting was assessed measuring the concentration of different methane samples. With this method, a minimum detectivity of 10 ppmv for the system configuration was estimated.
\end{abstract}

Keywords: Hollow-core photonic bandgap fibers, Near-infrared spectroscopy, Gas sensing, Methane.

\section{INTRODUCTION}

Optical gas spectroscopic systems are attractive for gas detection since they provide high spectral resolution, precise gas species identification and possibility of remote and distributed measurements [1]. Recently, a new class of optical fibres, the hollow-core photonic bandgap fibers (HC-PBFs), with unique properties that make them suitable for gas sensing has emerged [2-4]. HC-PBFs rely on a guiding mechanism in which light is trapped in a low-index core by a photonic bandgap existing in the cladding. When the hollow core of the fiber is filled with gas, long interaction lengths between light and the gas confined in the core can be achieved, thus enabling sensitive devices. Furthermore, HC-PBFs are particularly interesting for their possibilities of integrability and compactness [5]. For these reasons, in the past few years, gas-filled HC-PBFs have been widely investigated in applications such as gas detection [6-8], high-resolution spectroscopy experiments [9-11], wavelength locking [12] and nonlinear-optics [13].

Monitoring methane $\left(\mathrm{CH}_{4}\right)$ concentration is essential in many industrial, environmental, biomedical and safety applications. Methane strongest absorption lines are located in the mid-infrared spectrum, around $3300 \mathrm{~nm}$. However, light sources and detectors in this region are expensive and cumbersome to operate. Alternatively, in the near infrared region, sources and detectors are available at low-cost. For that reason, methane diode laser-based sensors have been typically developed in the near-infrared region, specifically, at $2 v_{3}$ band, located at $1670 \mathrm{~nm}$ [14]. With the advent of HC-PBFs, new prospects for methane detection have been opened. However, until recently, HC-PBFs have only been available in the weakly absorbing band of $1300 \mathrm{~nm}[6,8]$ and in the mid-infrared band [7].

In this paper, we report the application of a 1670-nm band HC-PBF to the detection of methane. This region benefits from the fully developed lasers and detectors available in this wavelength range, in addition to the possibility of remote sensing. Methane concentration was measured by targeting the R(6) absorption line at $1645 \mathrm{~nm}$ (near $6077 \mathrm{~cm}^{-1}$ ) since it is the strongest feature in this region. This is a manifold of six transitions strongly broadened at our experimental conditions, hence overlapped in a single line. This profile was fitted with a multiline algorithm to recover the lineshapes

Photonic Crystal Fibers II, edited by Kyriacos Kalli, Waclaw Urbanczyk,

Proc. of SPIE Vol. 6990, 69900W (2008)

0277-786X/08/\$18 $\cdot$ doi: $10.1117 / 12.780587$

Proc. of SPIE Vol. $699069900 \mathrm{~W}-1$ 
of each individual transition. This technique has been validated by measuring the concentration of different methane samples. The sensitivity (minimum detection limit) of this arrangement is also estimated. Finally, the filling dynamics of the HC-PBF is also studied.

\section{ABSORPTION SPECTROSCOPY THEORY}

The transmittance, $T_{v}$, at a frequency $v\left(\mathrm{~cm}^{-1}\right)$ is defined as the amount of light transmitted through an absorbing gas, $I_{t}$, with respect to the incident light, $I_{o}$. This value is exponentially related to both the properties of the gas species, through the spectral absorption coefficient, $k_{v}\left(\mathrm{~cm}^{-1}\right)$, and the distance the light travels or path length, $\mathrm{L}(\mathrm{cm})[1]$ :

$$
T_{v}=\frac{I_{t}}{I_{o}}=\exp \left(-k_{v} \cdot L\right)
$$

The spectral absorption coefficient, $k_{v}$, is given by [15]:

$$
k_{v}=\sum_{i=1}^{N} \operatorname{Px} S_{i} \phi_{i}\left(\Delta v_{i}, v-v_{0 i}\right)
$$

Here, $N$ represents the number of transitions within a manifold, $i$ a specific transition, $P$ (atm) the total pressure of the medium, $x$ the mole fraction of the gas species (related to the gas concentration), $S_{i}\left(\mathrm{~cm}^{-2} \mathrm{~atm}^{-1}\right)$ the linestrength for transition $i$ and $\phi_{i}\left(\Delta v_{i}, v-v_{0 i}\right)(\mathrm{cm})$ the lineshape function for transition $i$ centered at $v_{0 i}$ with spectral width, $\Delta v_{i}$. This lineshape function is represented by a Gaussian profile when the effects of line broadening are due to thermal motion (Doppler broadening). The width of the lineshape is then described by the Doppler width, $\Delta v_{D}$. Alternatively, collision effects, due to pressure, give rise to a Lorentzian lineshape function (collisional broadening), characterized by the collisional width parameter, $\Delta v_{C}$. When both effects are significant, the resultant lineshape function is typically addressed using a Voigt profile.

A more convenient way of representing Eq. 1 is by means of the absorbance, $\mathrm{A}_{v}$, as the dependence with the gas parameters is linear:

$$
A_{v}=-\ln \left(\frac{I_{t}}{I_{o}}\right)=k_{v} \cdot L=P L x \sum_{i=1}^{N} S_{i} \phi_{i}\left(\Delta v_{i}, v-v_{0 i}\right)
$$

\section{EXPERIMENTAL SETUP}

A scanned-wavelength direct absorption scheme was used to measure methane concentration. In this case, the laser frequency is tuned over a selected absorption line to measure its absorbance. The experimental setup devised for the detection of methane with a HC-PBF is depicted in Fig. 1. As can be seen, the measurement setup is similar to conventional direct absorption schemes, except for the replacement of the bulk gas cell with a HC-PBF.

The HC-PBF used in the experiments was provided by the Optoelectronics Research Centre at Southampton. A picture of the hollow-core and the surrounding microstructured cladding can be seen as an inset in Figure 1. The fiber has a core diameter of $12 \mu \mathrm{m}$, composed of 7 missing unit cells, and an overall cross section of $230 \mu \mathrm{m}$ diameter. The losses of the fiber are reported to be $100 \mathrm{~dB} / \mathrm{km}$. A piece of 5.1-m-long HC-PBF was used as gas cell and placed inside a vacuum chamber. Light from a tunable laser (Santec TSL-210V, spectral linewidth $1 \mathrm{MHz}$, i.e. $3 \cdot 10^{-5} \mathrm{~cm}^{-1}$ ) was launched to the HC-PBF through a single-mode fiber (SMF). A gap was left between the SMF and the HC-PBF to allow the gas access the hollow core of the fiber. The other end of the HC-PBF was spliced to a SMF and connected to a power meter (Agilent 8163A). A pump was used to evacuate the air from the chamber and a pressure gauge monitored the pressure conditions in the chamber. 


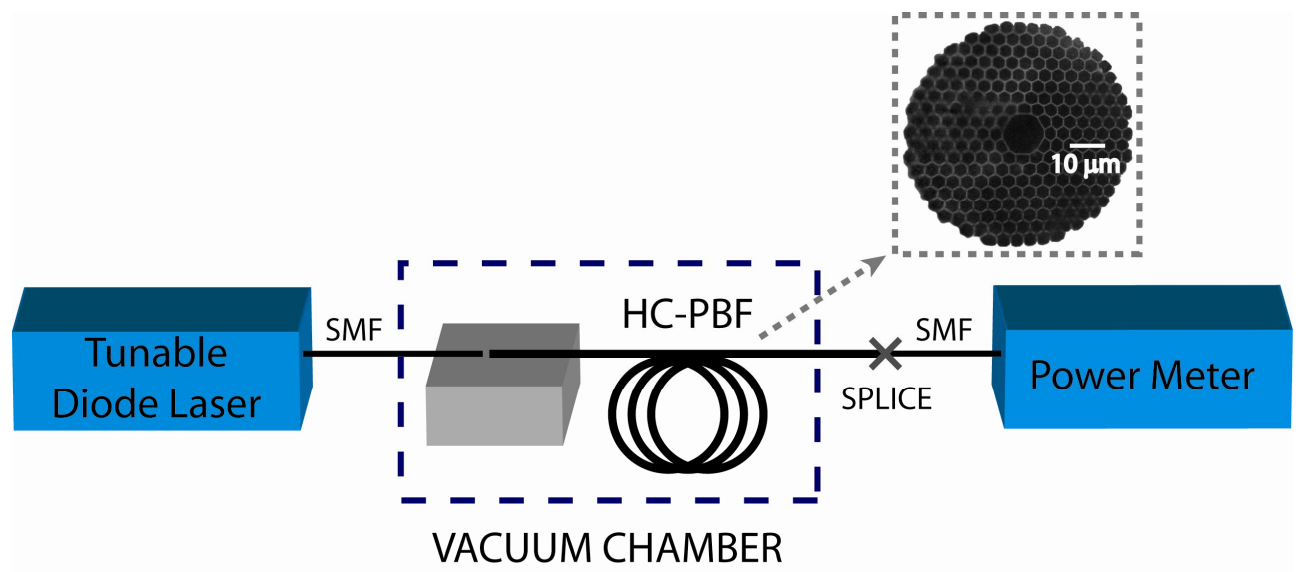

Fig. 1. Experimental setup for the detection of methane with a 5.1-m-long HC-PBF as gas cell. Shown as an inset is the microscope image of the HC-PBF cross section.

The transmission spectrum of the HC-PBF was measured to check the suitability of the HC-PBF for methane detection. The normalized transmission window is illustrated in Fig. 2. The transmission bandgap sharply starts at $1375 \mathrm{~nm}$ and goes beyond $1700 \mathrm{~nm}$ (until $1850 \mathrm{~nm}$ according to specifications). The inset of Figure 2 shows a zoomed section of the transmission of the fiber for the $2 v_{3}$ absorption band of methane. As can be seen, the fiber shows minimum losses in this wavelength range, which makes it feasible its application to methane detection.

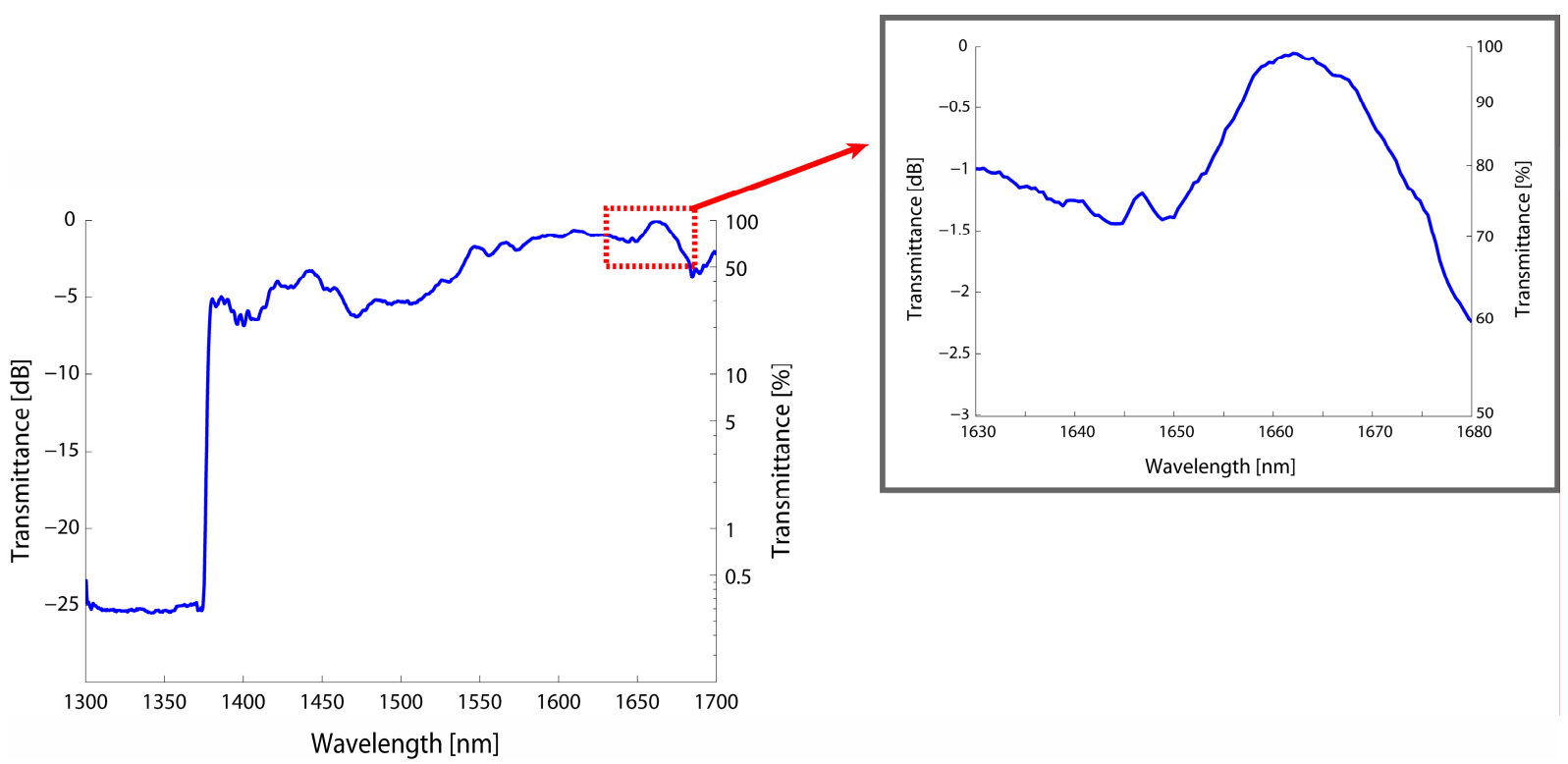

Fig. 2. Normalized spectral transmission of the HC-PBF used in the experiments. Shown as an inset is a zoomed section of the spectral transmission of the fiber for the $2 v 3$ absorption band of methane.

\section{RESULTS AND DISCUSSION}

\subsection{Methane transmission spectrum}

Methane $2 v_{3}$-band transmission spectrum for a calibrated concentration of $18750 \mathrm{ppmv}$ methane in air was measured using the system sketched in Fig. 1. The spectrum window was scanned from $1625 \mathrm{~nm}$ to $1680 \mathrm{~nm}$ with a resolution of $0.025 \mathrm{~nm}$. The resulting spectrum, illustrated in Fig. 3, agrees with HITRAN database absorption lines [16]. R, Q and P 
branches at $2 v_{3}$ band are clearly seen and labelled in the figure. The $\mathrm{R}(6)$ manifold at $1645 \mathrm{~nm}$, indicated in the figure, was chosen for concentration measurements as it shows the best signal-to-noise ratio in the band.

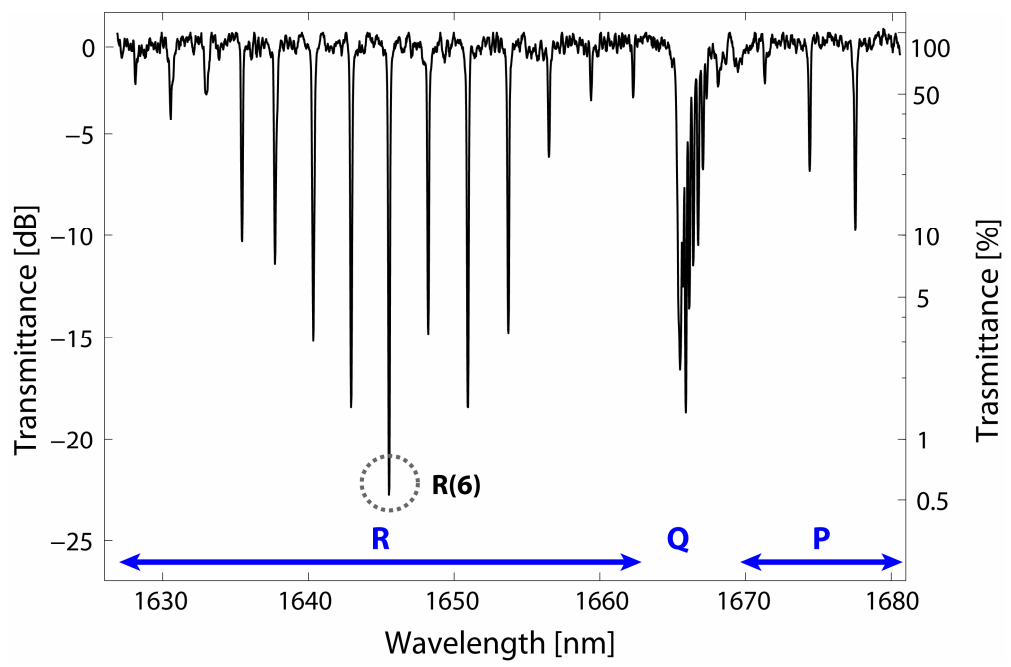

Fig. 3. Transmission spectrum of methane measured at room temperature and a relative pressure of 1 bar with a methane concentration of $18750 \mathrm{ppmv}$ in air. The scanned resolution was $0.025 \mathrm{~nm}$ from $1625 \mathrm{~nm}$ to $1680 \mathrm{~nm}$ (limited by the light source). P, Q and R branches are labelled. The R(6) absorption line, chosen for concentration measurements, is indicated with a circle.

\subsection{Filling dynamics}

Using the arrangement of Fig. 1, the filling dynamics of the fiber was studied as it highly influences the response time of the sensor. Although the chamber can be filled with gas in a few seconds, the time needed for the gas to diffuse along the fiber is strongly dependent on the gas species, the length of the fiber and the pressure [6]. To evaluate the filling process, the power of the light transmitted through the HC-PBF was monitored at the R(6) line when the chamber was filled with gas at a relative pressure of 1 bar. The light transmission as a function of time follows an exponential decay with 7 minutes required to reach a steady state (Fig. 4). This filling time might be a major limit the response time of the sensor. However, this gas diffusion process could be speeded up by opening access points to the hollow-core through the cladding $[17,18]$ or by using multi-coupling gaps instead of a single piece of fiber $[19,20]$. We are currently carrying out further research to reduce this filling time.

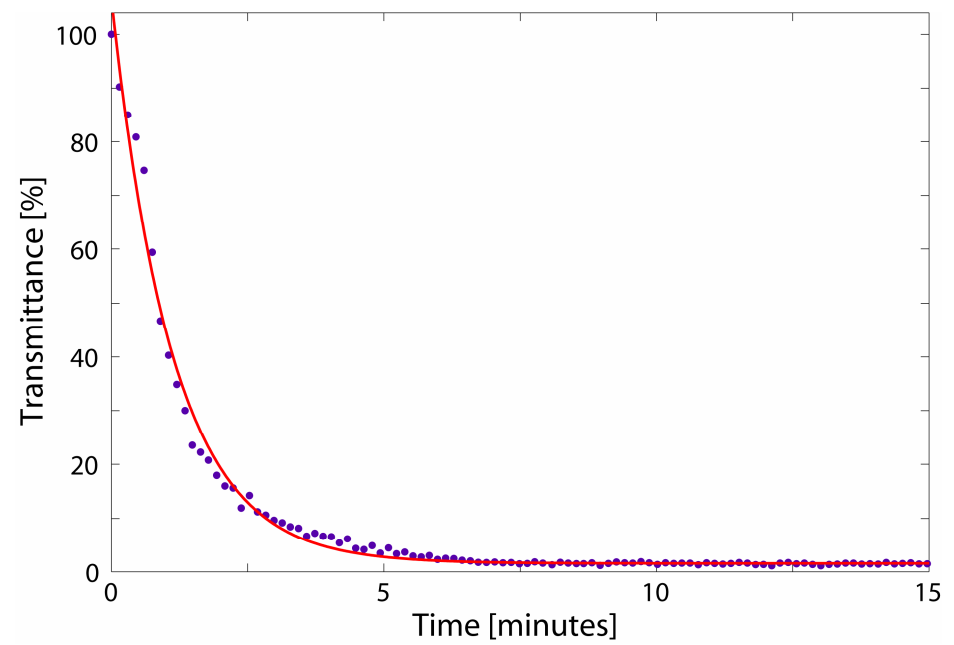

Fig. 4: Measured normalized transmittance at the $R(6)$ absorption line as a function of time when the vacuum chamber is filled with a 18750 ppmv concentration of methane at a relative pressure of 1 bar and room temperature. Dots represent the experimental data and solid red line the exponential fitting. 


\subsection{Data analysis}

The $\mathrm{R}(6)$ absorption line, selected for concentration measurements, is actually the overlapping resultant of a set of different transitions, a manifold. Specifically, it is comprised of six individual transitions, as listed in Table 1 [16]. At our experimental conditions (room temperature, 1 bar of relative pressure), these transitions are strongly broadened and overlapped into a single spectral line. A multiline fit model will therefore be used to take into account and recover all the transitions within the manifold. In our case, the effect of thermal broadening is negligible compared to the broadening due to collisional effects. Thus, the lineshape function of the individual transitions follows a Lorentzian function [21].

Table 1. Individual transitions of the $\mathrm{R}(6)$ manifold $^{a}$

\begin{tabular}{ccc}
\hline Transition & $v_{0}\left(\mathrm{~cm}^{-1}\right)$ & $\mathrm{S}\left(\mathrm{cm}^{-2} \cdot \mathrm{atm}^{-1}\right)$ \\
\hline 1 & 6076.928 & $1.192 \cdot 10^{-2}$ \\
2 & 6076.935 & $1.899 \cdot 10^{-2}$ \\
3 & 6076.954 & $3.000 \cdot 10^{-2}$ \\
4 & 6077.028 & $1.768 \cdot 10^{-2}$ \\
5 & 6077.046 & $1.847 \cdot 10^{-2}$ \\
6 & 6077.063 & $3.050 \cdot 10^{-2}$ \\
Manifold & & $1.276 \cdot 10^{-1}$ \\
\hline${ }^{a}$ From J. Quant. Spectrosc. Radiat. Transfer 96, 139(2005).
\end{tabular}

In this way, and according to Eq. 3, to fit the R(6) absorption profile, three parameters per transition are necessary, i.e. $S_{i}$, $\Delta v_{i}$ and $v_{0 i}$. Following the model used in [15], some simplifications can be made to reduce the number of parameters. The collisional broadening can be considered to be equal for all the transitions in the manifold. Moreover, at room temperature, the individual linestrengths of each transition, $S_{i}$, can be expressed in terms of the total linestrength of the manifold, $S_{t o t}$, and a relative contribution, $\omega_{i}$, of each transition to the total linestrength. Furthermore, the central frequency of each transition, $v_{0 i}$, is represented by an arbitrary central frequency, $v_{0}$, and the wavenumber spacing of each transition $i$ to $v_{0}, \delta v_{i}$, as follows:

$$
v_{0 i}=v_{0}+\delta v_{i}
$$

Both $\omega_{i}$ and $\delta v_{i}$ parameters are calculated from HITRAN database [16]. This finally yields to a 3-parameter model $\left(S_{t o t}\right.$, $v_{0}, \Delta v_{C}$, which is a much easier way to handle the overlapped transitions within the manifold:

$$
A_{v}=P L x \sum_{i=1}^{6} S_{t o t} \omega_{i} \phi_{i}\left(\Delta v, v-v_{0 i}\right)
$$

The R(6) absorption lineshape was first analyzed to obtain important information such as the absorption linestrength and broadening width. Therefore, the three parameters of the model were determined and compared to the theoretical values provided by HITRAN database. For that purpose, we calculated the absorbance of the R(6) manifold from the measured transmitted intensity and the incident intensity, using Eq. 3. These data points are plotted as dots in Fig. 5 (upper). They were obtained using a $18750 \mathrm{ppmv}$ sample of methane in air at room temperature and a relative pressure of 1 bar. The absorbance measurements were then non-linear least-squared fitted with a Levenberg-Marquardt algorithm using the model described in Eq. 5. The resultant 6-line Lorentzian fit is represented as a red solid curve. The residuals (bottom) are calculated as the difference between the data and the curve fit. As can be observed, the fit and the data show a very good correspondence. This Lorentzian fit provides the values of the three parameters of the model (i.e. $\mathrm{S}_{\mathrm{tot}}=1.304 \cdot 10^{-1}$ $\mathrm{cm}^{-2} \cdot \mathrm{atm}^{-1}, v_{0}=6076.930 \mathrm{~cm}^{-1}, \Delta v=0.0849 \mathrm{~cm}^{-1}$ ), which are later used to obtain the gas concentration. Furthermore, the contributions from the individual transitions are illustrated as broken curves. 


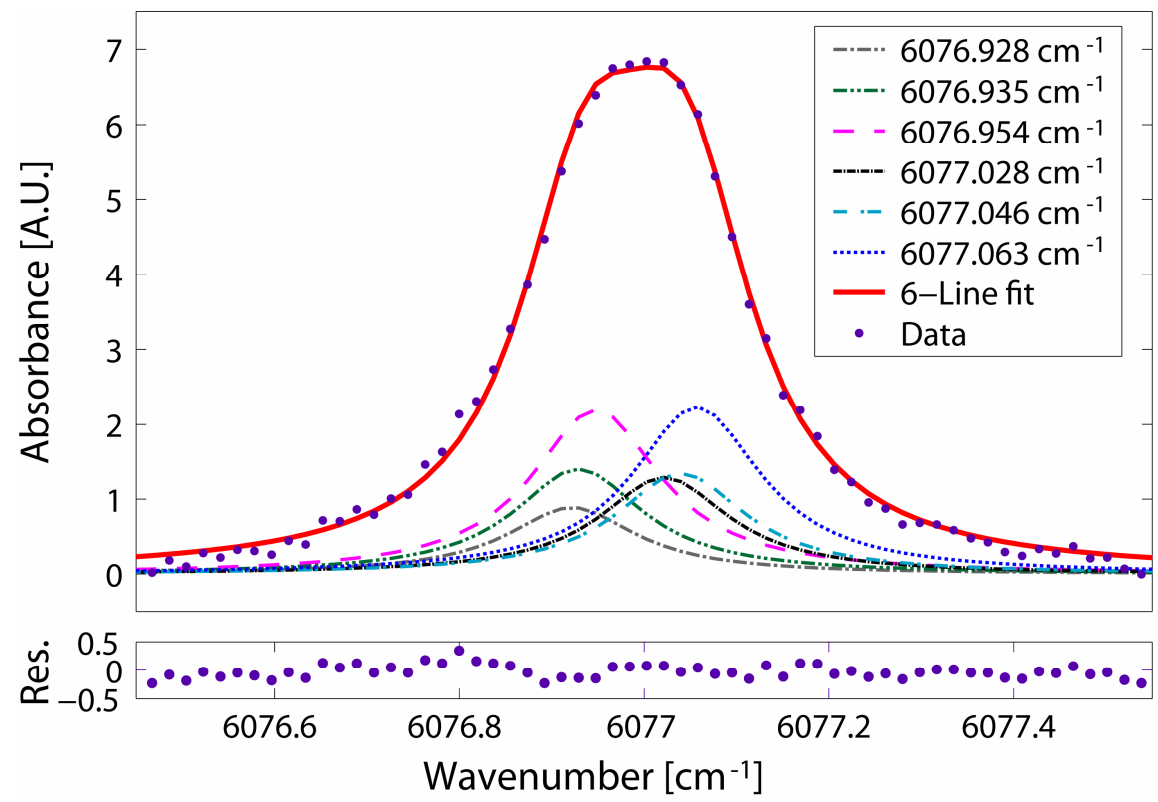

Fig. 5. 6-Line best Lorentzian fit (solid red curve) for $\mathrm{R}(6)$ manifold near $6077 \mathrm{~cm}^{-1}$ at $\mathrm{T}=296 \mathrm{~K}$, relative pressure of 1 bar and methane concentration of $18750 \mathrm{ppmv}$ (upper). Dots representing the experimental points and broken lines the contributions of each of the six transitions of the $\mathrm{R}(6)$ absorption line. The residual (bottom) is calculated as the difference between the data (dots) and the multiline best-fit Lorentzian profile (solid red curve).

\subsection{Sensitivity of the system}

To evaluate the sensitivity of the arrangement, the HC-PBF was filled with a concentration of 750 ppmv of methane in air at a relative pressure of 1 bar. The curve fitting of the $\mathrm{R}(6)$ feature was performed using the procedure described before and the result is shown in Fig. 6. Etalon fringes due to cladding modes of the HC-PBF were present during this experiment but were reduced by 10 sample averaging. The detection limit was extrapolated as the concentration obtained with a SNR equal to 1 . In this case, the SNR of the system was 75 , which yields to a detection limit of 10 ppmv.

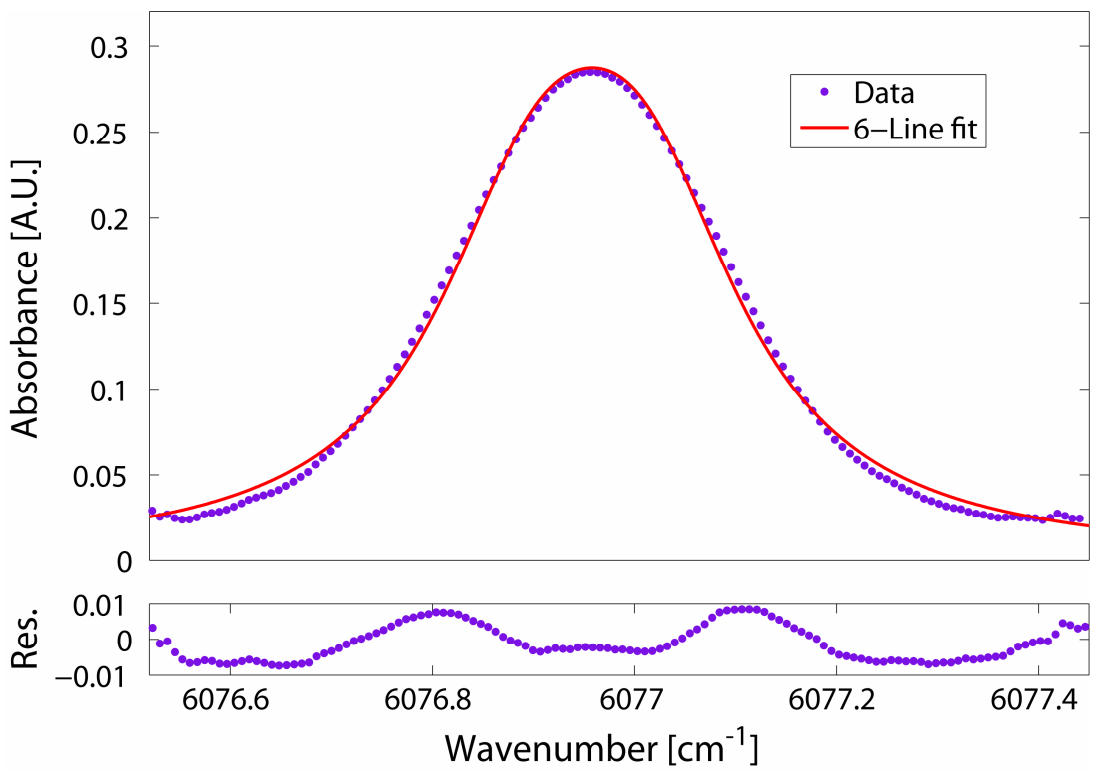

Fig. 6. 6-Line best Lorentzian fit for the $\mathrm{R}(6)$ manifold near $6077 \mathrm{~cm}^{-1}$ at $\mathrm{T}=296 \mathrm{~K}$, relative pressure of 1 bar and methane concentration of $750 \mathrm{ppmv}$ (upper) and residual (bottom). The SNR of the system is 75 which gives rise to a detection limit of 10 ppmv. 


\subsection{Concentration measurements}

To validate this technique, the multiline algorithm was applied to estimate the concentration for a set of different methane calibrated samples. For scanned-wavelength detection schemes, the concentration can be calculated as follows:

$$
x=\frac{\text { Area }}{P L x \sum_{i=1}^{6} S_{\text {tot }} \omega_{i} \phi_{i}\left(\Delta v_{i}, v-v_{0 i}\right)}
$$

Here, the Area is the integrated absorbance of the gas through the R(6) absorption line and $S_{t o t}, v_{0}$ and $\Delta v$ are the parameters of the model obtained previously.

Fig. 7 shows a set of recovered methane concentration using this procedure (dots), as a function of the measurement number, plotted against the calibrated methane concentrations (dashed red line). A very good agreement is shown between the experimental results and the calibrated samples, which clearly demonstrates the repeatability of the experiment.

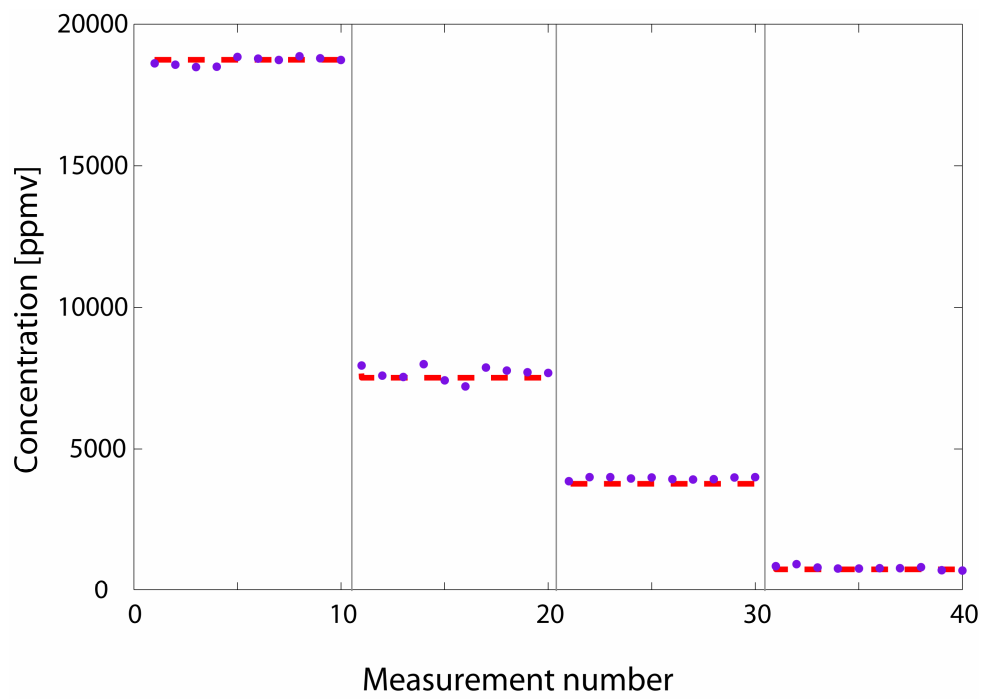

Fig. 7. Concentration measurements of methane estimated with the 6-line fit algorithm. Dots represent the experimental data and dashed lines the calibrated value. Each block represents a different set of methane concentrations

\section{CONCLUSIONS}

In this paper we have demonstrated the application of a 1670-nm band HC-PBF to the detection of methane. This band, despite having relatively weak absorbing lines, benefits from the long path lengths achievable with HC-PBF technology. The $\mathrm{R}(6)$ absorption line (near $1645 \mathrm{~nm}$ ) was targeted because it showed the best SNR in the band. At our experimental conditions, collisional broadening made impossible the identification of the individual transitions that comprise the R(6) manifold. A multiline model was therefore used to fit the manifold. The fit allows us to infer the methane concentration in air with a minimum detection limit of 10 ppmv. Furthermore, the diffusion time for the gas along the fiber was calculated to be $\sim 7$ minutes, which might limit the response time of the sensor. Further research is still required to reduce the diffusion time of gas inside HC-PBFs.

\section{ACKNOWLEDGMENTS}

The authors acknowledge financial support for this work provided by the Spanish Government's Ministry of Science and Technology via the TEC'2005-08218-C02-02 and TEC'2007-67987-C02-01 projects. We also thank Prof. Rebolledo, from the University of Zaragoza, for his assistance with the arc fusion splicing procedure. 


\section{REFERENCES}

[1] Lopez-Higuera, J. M., [Handbook of Optical Fibre Sensing Technology], Ed. John Wiley \& Sons, (2002).

[2] Birks, T. A., Roberts, P. J., Russel, P. St. J., Atkin, D. M. and Shepherd, T. J., "Full 2D photonic band gaps in silica/air structures," Electron. Lett. 31, 1941-1943 (1995).

[3] Cregan, R. F., Mangan, B.J., Knight, J. C., Birks, T.A., Russel, P. St. J., Roberts, P. J. and Allan, D. C., "Singlemode photonic band gap guidance of light in air," Science 285, 1537-1539 (1999).

[4] Benabid, F., "Hollow-core photonic bandgap fibre: new light guidance for new science and technology," Phil. Trans. R. Soc. A 364, 3439-3462 (2006).

[5] Benabid, F., Couny, F., Knight, J. C., Birks, T. A. and Russell, P. St. J., "Compact, stable and efficient all-fiber gas cells using hollow-core photonic crystal fibers," Nature 434, 488-491 (2005).

[6] Ritari, T., Tuominen, J., Ludvigsen, H., Petersen, J. C., Sorensen, T., Hansen, T. P. and Simonsen, H. R., "Gas sensing using air-guiding photonic bandgap fibers," Opt. Express 12, 4080-4087 (2004).

[7] Kornaszewski, L. W., Gayraud, N., Stone, J. M., Macpherson, W. N., George, A. K., Knight, J. C., Hand, D. P. and Reid, D. T., "Mid-infrared methane detection in a photonic bandgap fiber using a broadband optical parametric oscillator," Opt. Express 15, 11219-11224 (2007).

[8] Cubillas, A. M., Lazaro, J. M., Silva-Lopez, M., Conde, O. M., Petrovich, M. N. and Lopez-Higuera, J. M., "Methane sensing at 1300-nm band with a hollow-core photonic bandgap fiber as gas cell," Electron. Lett. (to be published) (2008).

[9] Henningsen, J., Hald, J. and Petersen, J .C., "Saturated absorption in acetylene and hydrogen cyanide in hollow-core photonic bandgap fibers," Opt. Express 13, 10475-10482 (2005).

[10] Thapa, R., Knabe, K., Faheem, M., Naweed, A., Weaver, O. L. and Corwin, K. L., "Saturated absorption spectroscopy of acetylene gas inside large-core photonic bandgap fiber," Opt. Lett. 31, 2489-2491 (2006).

[11] Hald, J., Petersen, J. C. and Henningsen, J., "Saturated optical absorption by slow molecules in hollow-core photonic band-gap fibers," Phys. Rev. Lett. 98, 213902 (2007).

[12] Tuominen, J., Ritari, T., Ludvigsen, H. and Petersen, J. C., "Gas filled photonic bandgap fibers as wavelength references," Opt. Commun. 255, 272-277 (2005).

[13] Couny, F., Light, P.S., Benabid, F. and Russell, P. St. J., "Electromagnetically induced transparency and saturable absorption in all-fiber devices based on 12C2H2-filled hollow-core photonic crystal fiber," Opt. Commun. 263, 2831 (2006).

[14] Culshaw, B., Stewart, G., Dong, F., Tandy, C. and Moodie, D., "Fibre optic techniques for remote spectroscopic methane detection," Sens. Act. B 51, 25-37 (1998).

[15] Gharavi, M. and Buckley, S.G., "Diode laser absorption spectroscopy measurement of linestrengths and pressure broadening coefficients of the methane 2v3 band at elevated temperatures," J. Mol. Spectrosc. 229, 78-88 (2005).

[16] Rothman, L. S. et al., "The HITRAN 2004 molecular spectroscopic database," J. Quant. Spectrosc. Radiat. Transfer 96, 139-204 (2005).

[17] Van Brakel, A., Grivas, C., Petrovich, M. N. and Richardson, D. J., "Micro-channels machined in microstructured optical fibers by femtosecond laser," Opt. Express 15, 8731-8736 (2007).

[18] Hensley, C. J., Broaddus, D. H., Schaffer, C. B. and Gaeta, A. L., "Photonic band-gap fiber gas cell fabricated using femtosecond micromachining," Opt. Express 15, 6690-6695 (2007).

[19] Hoo, Y. L., Jin, W., Shi, C., Ho, H. L., Wang, D. N. and Ruan, S. C., "Design and modeling of a photonic crystal fiber gas sensor," App. Opt. 42, 3509-3515 (2003).

[20] Lazaro, J. M., Cubillas, A. M., Silva-Lopez, M., Conde, O. M., Petrovich, M. N. and Lopez-Higuera, J. M., "Methane sensing using multiple-coupling gaps in hollow-core photonic bandgap fibers," 19th International Conference on Optical Fibre Sensors (Perth, Australia) (2008).

[21] Nagali, V. and Hanson, R.K., "Design of a diode-laser sensor to monitor water vapour in high-pressure combustion gases," App. Opt. 36, 9518-9527 (1997).

[22] Webber, M. E., Kim, S., Sanders, S. T., Baer, D. S., Hanson, R. K. and Ikeda, Y., "In situ combustion measurements of $\mathrm{CO} 2$ by use of a distributed-feedback diode-laser sensor near 2.0 um," App. Opt. 22, 821-828 (2001). 